\section{Pneumokokken?} Impfen!

Die häufigsten Erre-
ger der ambulant er-
worbenen Pneu-
monie (CAP) sind Pneumokokken. Daher könnte die Impung für mulitmorbide Patienten lebensrettend sein. Die Häufigkeit und Schwere hänge, laut Prof. Adrian Gillissen, Kassel, vom Alter (> 65 Jahre) und Komorbiditäten wie z.B. Diabetes oder COPD ab. „20\% der Patienten mit CAP werden krankenhauspflichtig, 2 \% intensivpflichtig “, sagte Gillissen. Zwar hätten Ärzte „oft leichtes Spiel“ bei der Wahl des Antibiotikums, dennoch treten in Deutschland auch resistente Pneumokokken auf (1-5\%).

PneumoNews 2016/1

\section{Neue Asthma-Leitlinie}

Die Leitlinie der DGP und der DAL ist nicht final freigegeben, doch der Inhalt steht, laut Prof. Dr. Roland Buhl, Mainz. Auf Stufe 1 wird die Bedarfstherapie mit schnellwirkenden Beta-2-Mimetika (SABA) genannt. Hier sollte die Möglichkeit zur Dauertherapie mit niedrig dosierten inhalativen Kortikosteroiden (ICS) erwähnt werden. Ab Stufe 2 ist die ICS-Therapie in niedriger bis moderater Dosis Standard. Alternativ werden nur Leukotrien-Rezeptor-Antagonisten (nur Montelukast) genannt. Auf Stufe 3 wird zur Bedarfstherapie alternativ zu SABA mono

\section{Therapieversagen bei über 30-jährigen}

Daten der „Centers for Disease Control and Prevention" legen nahe, dass mit dem Altern sowohl Morbidität als auch Mortalität bei Asthmapatienten zunehmen. Doch bisher gab es keine Untersuchung zum Thema. Deshalb haben Pneumologen und Forscher um Ryan M. Dunn von der Universität von Colorado, die Befunde von 1200 Patienten ausgewertet, die an zehn Studien des „Asthma Clinical Research Network“ zwischen 1993 und 2013 teilgenommen hatten. Mit jeden zusätzlichen fünf Jahren Lebensalter sei der Analyse zufolge die Wahrscheinlichkeit, nicht mehr auf die Asthmabehandlung anzusprechen, um 13 Prozent gestiegen, alle zehn Jahre um 27 Prozent.

PneumoNews 2015/4: 52

auch die Kombination ICS/Formoterol empfohlen. Für die Stufe 4 wird primär die Kombination ICS/LABA plus Tiotropium genannt, Alternativen sind Kombinationen mit hochdosiertem ICS +/-LABA, +/-Tiotropium oder +/-LTRA, ggf. auch eine 4erKombi, so Buhl. Auf Stufe 5 werden additiv anti-IgE-Antikörper bei allergischem bzw. anti-IL-5-Hemmer bei eosinophilem Asthma (Grenzwert: $\geq 300 / \mu l$ ) empfohlen.

57. Kongress der Deutschen Gesellschaft für Pneumologie, Leipzig, März 2016; Klinisches Symposium: Pneumologische Leitlinien - Möglichkeiten und Grenzen

\title{
Krebsmedikamente gegen die PAH?
}

„Die pulmonale arterielle Hypertonie ist eine proliferative, nicht nur eine vasokonstriktorische Erkrankung“, erläuterte PD Dr. Hans-Jürgen Seyfarth, Leipzig. Dass eine Neoplasie stattfindet, lässt sich auch in FDG-PET-Untersuchungen erkennen. Bislang stand therapeutisch die Vasodilatation im Vordergrund, neue Forschungsarbeiten verfolgen jetzt den Ansatz der Antiproliferation. So konnten mit dem Tyrosinkinaseinhibitor Imatinib laut Seyfarth ,beeindruckende Ergebnisse“ erzielt werden mit deutlichem Abfall des pulmonal-arteriellen Drucks und besserer Herzfunktion. Auf- grund ernster Nebenwirkungen erhielt die Substanz für die PAH keine Zulassung. Der mTOR-Inhibitor Everolimus konnte in einer kleinen Studie mit zehn Patienten innerhalb von sechs Monaten die hämodynamische Situation verbessern. Der Serotoninrezeptor-Antagonist Tergurid, der in Zellkulturen die Proliferation hemmte, zeigte in einer klinischen Studie mit $16 \mathrm{~Pa}$ tienten gute Erfolge.

57. Kongress der Deutschen Gesellschaft für Pneumologie, Leipzig, März 2016; Messestadtsymposium „Innovative Pneumologie: Therapie"

\section{Bei Lungenembolie zählt jede Minute!}

Atemnot und Thoraxschmerz lassen in erster Linie an einen Herzinfarkt denken, doch kann sich dahinter auch eine Lungenembolie verbergen! Um der richtigen Differenzialdiagnose auf die Spur zu kommen, raten Experten, Risiko-Scores anzuwenden. Verläuft die Embolie fulminant, liegt ein echter Notfall vor und dann zählt jede Minute. Was in diesem Fall zu tun ist, lesen Sie neben weiteren spannenden Themen im Schwerpunkt "Atemwegserkrankungen“!

Claudia Daniels Redakteurin

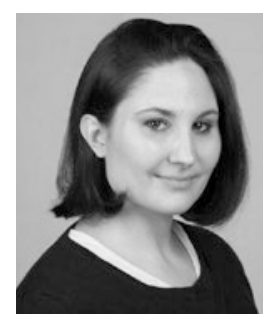

\section{Konservativer Start der AB-Therapie}

Laut einer retrospektiven 10-Jahres-Untersuchung hat sich bei akut exazerbierten COPD-Patienten mit Infektion der unteren Atemwege der Einsatz von Breitband-Antibiotika wie Aminoglykosiden oder Ciprofloxacin verdreifacht, berichtete Prof. Dr. Rainer Hauck, Altötting. Dies habe häufig irrationale Gründe. Ein konservativer Start der Antibiotikatherapie bedeute keine Verschlechterung der Prognose. Bei Patienten mit leichter bis mittelgradiger ambulant erworbener Pneumonie (CAP) ist eine BetaLaktam-Monotherapie, einer Kombi von Beta-Laktam-Antibiotikum / Makrolid bzw. eine Therapie mit einem Fluorchinolon, nicht unterlegen, betonte Prof. Dr. Stefan Krüger aus Düsseldorf. In einer Studie bei 2283 CAP-Patienten war die 90-TageMortalität mit 9-11\% in allen drei Gruppen ähnlich. Häufig besteht bei Patienten mit schweren AECOPD allerdings auch eine bakterielle Multiresistenz. Dazu können offenbar Glukokortikoide beitragen. Sie modulieren den Biofilm von Haemophilus influenzae (H.i.), einem häufigen Erreger von Atemwegsinfektionen und können so die Antibiotikatoleranz erhöhen, sagte Hauck.

57. Kongress der Deutschen Gesellschaft für Pneumologie, Leipzig, März 2016; Klinisches Symposium: Neues aus der Infektiologie 